\title{
A Watermarking Algorithm for 3D Videos Stream Based on Spatiotemporal Correlation
}

\author{
Li Wenfeng, Jiang Gangyi, Luo Ting \\ Faculty of Information Science and Engineering, Ningbo University, Ningbo China \\ liwenfengxie@foxmail.com, jianggangyi@126.com
}

Keywords:3D videos;Drift-level; Spatiotemporal correlation; Secret sharing; Matrix embedding

\begin{abstract}
When embed watermark into compressed video stream, distortion drift, including intraframe and inter-frame, is of the first important problem that must be taken into consideration. With the analysis of hierarchical b-picture (HBP) coding structure and drift-level mechanism, a new watermarking algorithm for 3D videos stream without distortion drift is proposed. Firstly the driftlevel of HBP coding structure is deeply investigated. Secondly, the spatiotemporal correlations of 3D videos are explored to increase the embedding capacity. Finally, the copyright information, which is preprocessed by secret sharing method, is embedded by matrix embedding method based on Error-correcting codes. Experimental results show that the proposed algorithm has higher embedding capacity and efficiency than the current algorithms.
\end{abstract}

\section{Introduction}

Three-dimensional (3D) videos technology is becoming increasingly popular, because it can provide stereoscopic perception with a strongly sense of realism and immersion to end users [1]. It will be useful for many new applications, such as 3D television (3DTV) and free-viewpoint television (FTV), which becomes more popular and will be largely commercialized in the near future [2]. Nevertheless, due to the growth of computer and internet technologies, 3D videos can be easily accessed, manipulated and tampered by using video processing tools. Therefore, it is a great challenge to ensure the copyright of $3 \mathrm{D}$ videos. Watermarking technology is regarded as one of effective approaches to resolve copyright protection [3]. Many researchers have begun to turn their attention to the 3D video watermarking [4].

To address the issues of image authenticity verification and integrity protection, a watermarking method for stereo image authentication based on stereo matching technique is proposed in [5]. But this method is lack of robustness to be used to actual application. A robust watermarking method for stereo-pair images based on unmatched block bitmap is proposed in [6], this algorithm exhibits greater robustness against malicious attacks and produces less distortion than existing methods do. Unlike stereo-pair images, 3D videos data is enormous, it often stored and transmitted in compressed format, the above mentioned methods which are focus on original stereo-pair images cannot be used to compressed video directly. In 2010, Joint Video Team (JVT) released the Multi-View Coding (MVC) [7] standard based on H.264/AVC, and wrote them as an appendix in the form of the latest H.264/AVC. JVT adopted the structure of hierarchical B-frames proposed in [8] which included the intra-prediction, the inter-prediction and the inter-view prediction. As it's a high efficient coding standard, H.264/MVC often used to compress 3D videos. As a result, researching watermarking methods that appropriate for this standard is highly desirable.

The watermarking methods that perform in H.264 compressed domain have been researched for many years [9]. For embedding watermark into compressed video, there are two approaches [10]: joint compression embedding and compressed domain watermarking. In the first category, the processes of watermarking and compression are performed jointly. The watermark can be added into inner feedback close-loop. Therefore, the error caused by watermark embedding does not propagate [11]-[12]. Nevertheless, when this method perform in the video that has been compressed, decompressing and recompressing the video stream are required, which are computationally expensive. In the second scenario, full decoding and recoding of the compressed video is not necessary. However this method may lead to error-drift if watermark embed improperly. If the drift distortions caused by watermarking do not eliminate, will cause error propagation. Therefore, 
solving the error-drift problem and preserving video quality is a research hotspot. At present there are two main technique to eliminate or control error-drift, first is the compensation method [13]-[14], and second is to embed watermark into the data which do not influence the following decoding[15][17].

Till now, few successful watermarking schemes in the H.264/MVC compressed stream have been reported in the open literature. In [18], a reversible video data hiding algorithm for MVC based on motion vector is proposed which can provide expected acceptable video quality of marked video and successfully achieve reversibility. Two more transparent data hiding scheme for 3D videos without error-drift are presented in [19] and [20], but the watermark capacity is small.

Based on the analysis given above, combining HBP coding structure features, we propose a new watermarking algorithm to 3D videos stream without error-drift and higher watermark capacity. Firstly the HBP coding structure is deeply investigated to analyze the drift-level. Secondly, in order to increase the embedding capacity, the spatiotemporal correlation is explored to find more appreciate candidate embedding locations. Finally, the copyright information, which is preprocessed by secret sharing method, is embedded by matrix embedding method based on error-correcting codes.

The remainder of this paper is organized as follows: First, we analyze the HBP coding structure with two viewpoints and its drift-level in Section II; the spatiotemporal correlations of $b_{4}$ frame in $3 \mathrm{D}$ videos are explored in Section III; our watermarking algorithm is presented in section IV; the experiment results are presented and the conclusion is given in section $\mathrm{V}$.

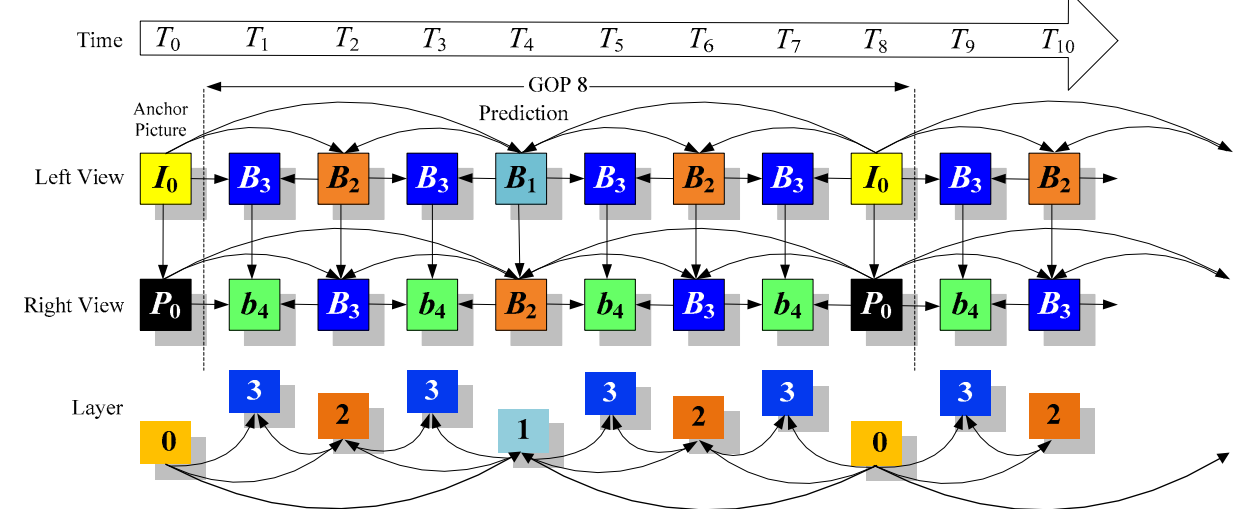

Fig.1. HBP structure with GOP size 8 with of two viewpoints

\section{The prediction structure of HBP and its drift-level analysis}

In this section, we briefly explain the HBP-based prediction structure. Moreover, we analyze the mechanism of error-drift and define the drift-level by its importance level to decoding. In general, in HBP-based coding structure the GOP size has to be set as power of 2, the order of the total layers $A$ with GOP size $B$ can be represented as $A=\log _{2} B$. Where layer $A$ is assigned as the highest layer which starts form 0 and is increased by 1.The typical HBP-based structure with GOP size 8 of two views is shown in Fig 1.. Obviously, in a GOP, there only one I frame and P frame which is the most important frame that will be referenced by all other B frames. The rest of pictures are bi-directionally predictive coded by referring to the pictures of lower layers. In the HBP structure, the lower layers are used as reference frame for higher layers. And as a main viewpoint, left view will be referenced by right view, the drift seriousness of left view is more than right view. Therefore we define the driftlevel as shown in Fig 1. The representation of drift-level contain of two parts, one is represent the frame type, the other is represent drift-level value e.g., $B_{3}$ represents the current frame type is B frame and its drift-level value is 3 . Obviously, the smaller the drift-level value of the frame, the more error-drift probability it will be. It's reasonable to embed watermark into the frames which drift-level are higher. Hence, $b_{4}$ frames are selected to embed watermark initially. 


\section{Spatiotemporal Correlation of 3D Videos}

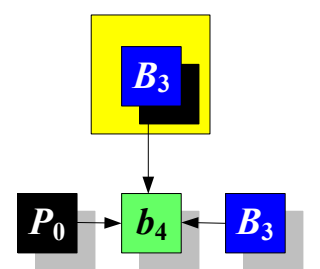

(a) $B_{3}$ frame in left view

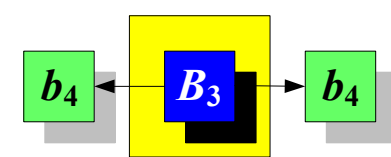

(b) $B_{3}$ frame in right view

Fig 2. $B_{3}$ frame in left view and right view

From above analysis, only the $b_{4}$ frame is drift-free in inter-view and temporal prediction structure. In HBP-based coding structure, $b_{4}$ frames will be allocated higher quantization step which make the probability of coded in skip modes largely increase. Therefore the redundant room for watermark embedding is very small. The drift-level of $B_{3}$ frame is higher than all other frames in a GOP except $b_{4}$ frame, so we want to select some appropriate $B_{3}$ frame to embedding. $B_{3}$ frames are exit either in left view or right view. $B_{3}$ frame in left view and right view are shown in Fig 2.(a).and Fig 2. (b), correspondingly. As shown in Fig 2. (a), $B_{3}$ frame in left view is only referenced by $b_{4}$ frame in the right view. $B_{3}$ frame in right view will be referenced by the other $b_{4}$ frames in right view as shown in Fig 2. (b). Further, we find that the number of $B_{3}$ frames in left view is double compare to right view. Hence, $B_{3}$ frames in left view are explored to embedding.

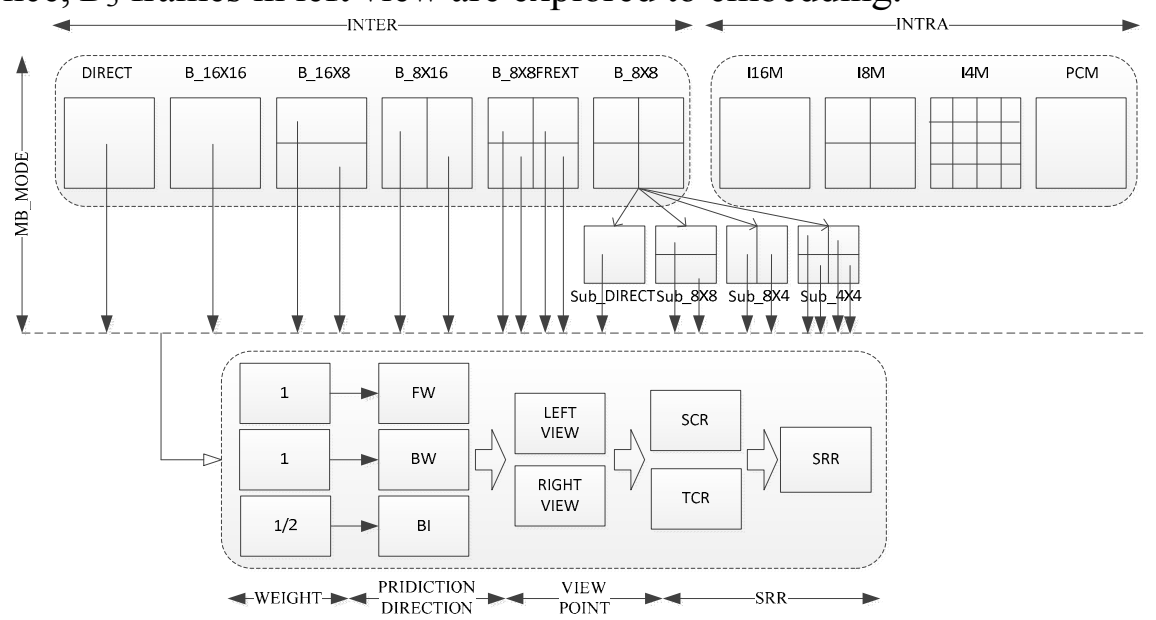

Fig.3. Illustration of SRR Analysis Process

Now, we analyze the spatiotemporal reference relationship (SRR) of $b_{4}$ frame to estimate the capacity payload of $B_{3}$ frame which located in left view. Ref [21] made a statistical analysis on the SRR in the original domain by computing the sum of absolutely difference (SAD) of motion/disparity match error. In this paper, we analyze SRR directly in compressed domain that are more exactly and efficient than in original domain. The frames referenced by $b_{4}$ frame can be divided into two parts, one is left view which represents the spatial correlation, and the other is right view which represents the temporal correlation. The ratio of spatial correlation (SCR) and temporal correlation (TCR) are used to represent the SRR of $b_{4}$ frame. As shown in Fig.3, there are DIRECT mode, Inter-MB modes (B16x16, B16x8, B8x16, B8x8Frext and B8x8) and intra-MB modes (I4MB, I8MB, I16MB and PCM) for a macro-block (MB) in B slices. Each $8 \times 8$ block of the B8x8 mode can be further sub-partitioned into smaller blocks and its prediction modes include Sub_DIRECT, Sub_B8x4, Sub_B4x8 and Sub_B4x4. The sub-partitioned blocks within a B8x8 block have the same reference frame. Because the smallest sub-block size is $4 \times 4$, we make a statistical analysis to SRR in 8 standard test video sequences which resolution is $640 \times 480$ based on $4 \mathrm{x} 4$ block. SCR and TCR are calculated as $S_{4}{ }^{*} W E I G H T /\left(S_{4}+T_{4}\right)$ and $T_{4}{ }^{*} \mathrm{WEIGHT} /\left(S_{4}+T_{4}\right)$, where $S_{4}$ is the total $4 \times 4$ blocks number that its reference frames located in the left view, $T_{4}$ is the total $4 \times 4$ blocks number that the reference frames located in the right view, the WEIGHT is determined by its prediction direction as shown in Fig 3.. The intra-MB is few in $b_{4}$ frames, so intra-MBs are ignored 
to calculate the SRR. The SRR is presented at table 1 in the form $<\mathrm{SCR}, \mathrm{TCR}>$. From table I we can find that TCR is more important compare to SCR. Therefore there are abundant spaces that suitable to embedding in $B_{3}$ frame which located in left view.

Table I Statistical results of SRR

\begin{tabular}{|l|l|l|}
\hline \multirow{2}{*}{ Video } & \multicolumn{2}{|c|}{ SRR } \\
\cline { 2 - 3 } & SCR(\%) & SRR(\%) \\
\hline Akko\&kayo & 4.63 & 95.37 \\
\hline Ballroom & 3.26 & 96.74 \\
\hline Crowd & 0.62 & 99.38 \\
\hline Exit & 0.33 & 99.67 \\
\hline Flamenco & 0.35 & 99.65 \\
\hline Objects & 0.02 & 99.98 \\
\hline Race & 2.85 & 97.15 \\
\hline Vassar & 0.35 & 99.65 \\
\hline
\end{tabular}

\section{The Proposed Watermarking Method}

We present our watermarking method in detail in this section. To begin with, we introduce two foundations of our scheme, i.e., secret sharing and matrix embedding.

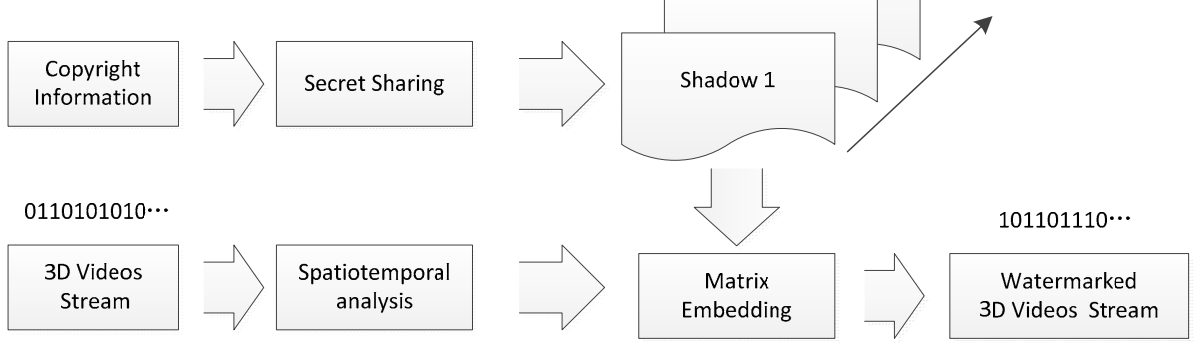

\section{A. Secret sharing}

Fig 4. Diagram of proposed method

Secret sharing is an important technique for sharing a secret to participants, each of which holds a portion of the secret. The secret can only be reconstructed when a certain number $t$ of members combine their shares together, while any combination with fewer than $t$ shares has no extra information about the secret than 0 shares. The first threshold scheme [22] was invented by Shamir and named as the Shamir threshold scheme. Shamir's $(t, n)$ threshold scheme is used widely in watermarking application. Because the watermarking capacity is not rich in $3 \mathrm{D}$ videos stream, we want to use Shamir's $(n, n)$ threshold scheme to divide the copyright information into $n$ shares and just to select a shadows to embed in the $3 \mathrm{D}$ videos stream. For lack of the $n-1$ shares, the unauthenticated user can't retrieve the copyright, so this scheme is security. Nevertheless, when use Shamir's $(t, n)$ threshold scheme to divide the information, each share's size is the same as the original information. Therefore, we must modify the method to divide the information without data size expansion. This paper takes a gray image as copyright information. Since the value of a gray image is between 0 and 255, the prime number $p=257$ is selected. The modified Shamir's $(n, n)$ threshold scheme presented as following steps:

\begin{tabular}{l}
\hline The modified Shamir's $(n, n)$ threshold scheme \\
\hline 1. Select a number $n$ \\
2. For each non-coincidence $n$ pixels of the gray image, a result $s_{n}(x)=s_{0}+\mathrm{s}_{1} x+\cdots$ \\
$+s_{k} x^{k-1}+\cdots+\mathrm{s}_{n-1} x^{n-1}(\bmod p)$ is calculate for a given $x$, where $s_{0}, \ldots, s_{k}, \ldots, s_{n-1}$ \\
correspond to the values of $n$ pixels. Change the value of $x$, and repeat this \\
step for $n$ times to generate $n$ different shadows. \\
3. Repeat step 2 until all the image shadows are generated.
\end{tabular}

Unlike original Shamir's $(n, n)$ threshold scheme, $s_{0}, \ldots, s_{k}, \ldots, s_{n-1}$ are correspond to the values of $n$ pixels that can constrain the shadow size un-expansion. 


\section{B. Matrix embedding based on correcting codes}

Matrix embedding (ME) [23] is an embedding method with high embedding efficiency, widely used for watermarking as the lower embedding distortions. Here we use ME combining with errorcorrection codes to watermarking. The correction codes [24] have been researched to achieve the high embedding efficiency. An example is now taken to show the matrix embedding method with Hamming codes. In general, ME is that $k$ secret bits $\boldsymbol{S}$ is embedded into $n$ cover bits $\boldsymbol{C}$, where $n=2^{k}$ 1, by modulating at most one cover bit. First the syndrome vector $\boldsymbol{Z}$ is calculated by $\boldsymbol{Z}=\left(\boldsymbol{H} \times \boldsymbol{C}^{T}\right)^{T} \oplus \boldsymbol{S}$, $\oplus$ is the exclusive-or operation and, $T$ is the transpose operation, $\boldsymbol{H}$ is the parity check matrix of $(n, n-k)$ Hamming code. Next, if the syndrome vector $\boldsymbol{Z}$ is a zero vector, then the cover bits needn't to modify; otherwise, find the $i$-th column of $\mathrm{H}$, where $1 \leq i \leq n$, that is equal to $\boldsymbol{Z}^{T}$. Then the cover bits $\boldsymbol{C}$ is calculate by $\boldsymbol{C}^{\prime}=\boldsymbol{e}_{\boldsymbol{i}} \oplus \boldsymbol{C}$, where $\boldsymbol{e}_{\boldsymbol{i}}$ is the $i$-th unit vector of length. At the receiving side, a receiver can easily extract the original information by $\boldsymbol{S}=\left(\boldsymbol{H} \times \boldsymbol{C}^{\boldsymbol{T}^{T}}\right)^{T}$. The embedding ratio of matrix embedding scheme is $p=k / n$, where $n=2^{k}-1$.

\section{The proposed watermarking algorithm}

The proposed watermarking method is shown in Fig.4. First the copyright information is preprocessed by secret sharing method to divide into $n$ shares. Then just a shadow is selected randomly to embedding by matrix embedding method.

Based on the analysis above, $B_{3}$ and $b_{4}$ frames are selected for candidate watermarking locations. The error-drift problem in inter-frame has been analyzed, now we analyze it in intra-frame briefly. In $B_{3}$ and $b_{4}$ frames there are two types $\mathrm{MB}$, one is intra-MB, and other is inter-MB.

In intra-MB compressed domain, mb type, code block pattern (CBP), prediction mode and integer DCT quantized coefficients (QDCT) are included. Since the mb_type, CBP and prediction direction are very important to decoding, the QDCT is selected to candidate embedding location only. As shown in Fig.5(a), to decode a $4 \times 4$ block in intra-MB, the adjacent pixels $A-M$ in the top left blocks are needed. Fig.5.(b) and (c) shows that the changed pixels will cause error-drift to its bottom right block. The decoding of $8 \times 8$ block in intra-MB is similar to $4 \times 4$ block. Hence, we use the method 1 that similar to ref [20] and [25] to add watermark into intra-MB without drift. The related special QDCT matrixes that can be used to embed without drift are :

$$
\boldsymbol{W}_{4}=\left[\begin{array}{cccc}
1 & 0 & -1 & 0 \\
0 & 0 & 0 & 0 \\
-1 & 0 & 1 & 0 \\
0 & 0 & 0 & 0
\end{array}\right] \boldsymbol{W}_{8}=\left[\begin{array}{cccccccc}
1 & 0 & 0 & 0 & -1 & 0 & 0 & 0 \\
0 & 0 & 0 & 0 & 0 & 0 & 0 & 0 \\
0 & 0 & 0 & 0 & 0 & 0 & 0 & 0 \\
0 & 0 & 0 & 0 & 0 & 0 & 0 & 0 \\
-1 & 0 & 0 & 0 & 1 & 0 & 0 & 0 \\
0 & 0 & 0 & 0 & 0 & 0 & 0 & 0 \\
0 & 0 & 0 & 0 & 0 & 0 & 0 & 0 \\
0 & 0 & 0 & 0 & 0 & 0 & 0 & 0
\end{array}\right] .
$$

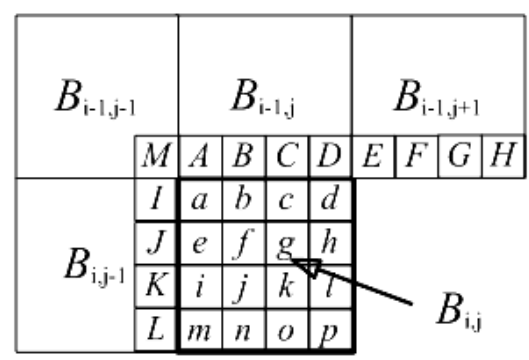

(a) Current $4 \times 4$ sub-block and its reference pixels

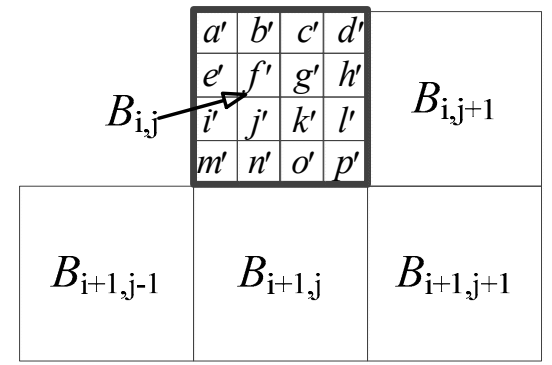

(b) Watermarked $4 \times 4$ sub-block 


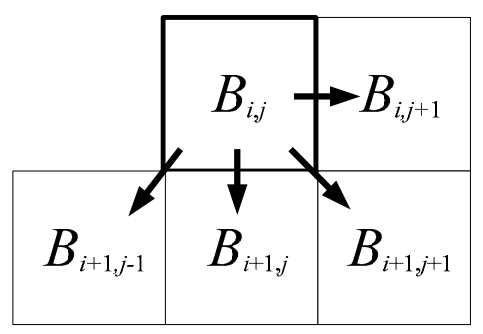

(c) Drift direction

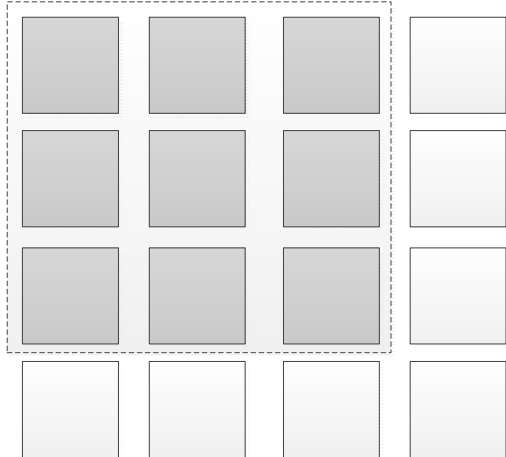

(d) Top left sub-blocks of a MB

Fig.5. Illustration of intra-frame drift

Inter-MB compressed domain consists of mb type, reference index (REF), motion vector deference (MVD) and QDCT. The mb type, REF and MVD are not considered to embedding as these data are crucial to decoding. As shown in Fig 5.(d), in order to prevent intra-frame error-drift, nine of the $4 \times 4$ blocks in the top left of the MB are selected to embedding in method 2 . The rest blocks of the MB are embedding in method 1 . If the transform size is $8 \times 8$, just one block in the top left is selected. The method 1 and method 2 are described as follow:

\section{Method 1}

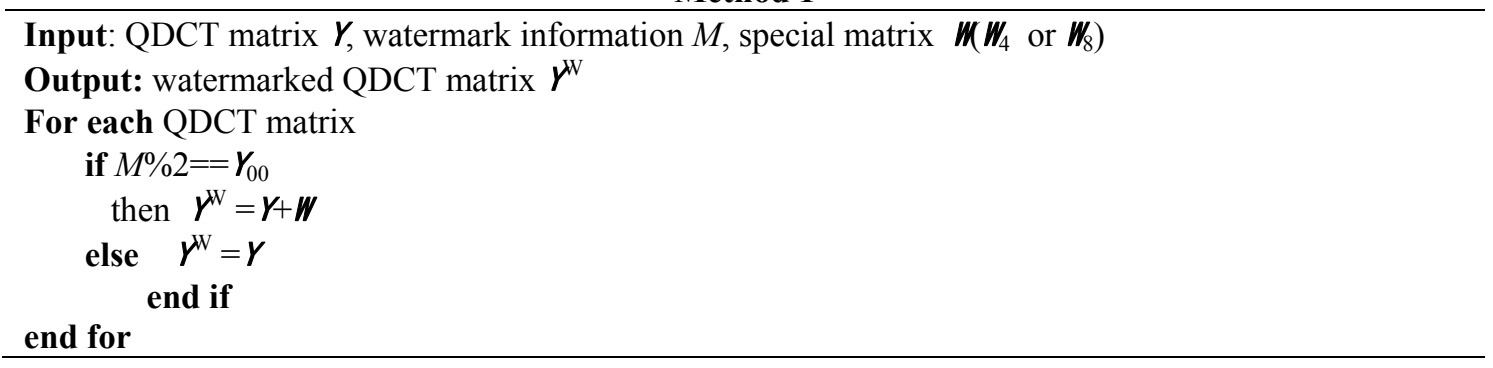

\section{Method 2}

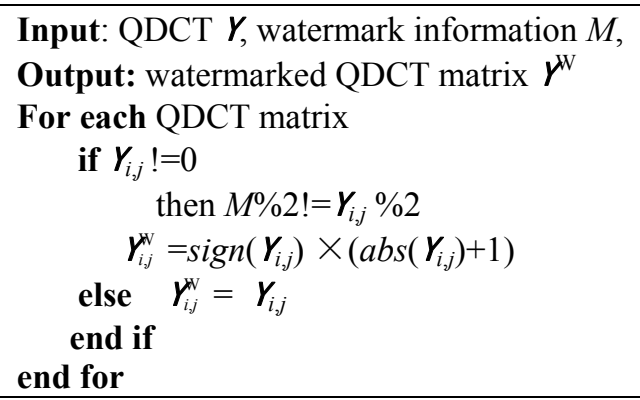

We have analyzed the intra-frame drift problem above, now we describe the proposed algorithm as follow:

\section{Proposed algorithm}

1. Encrypt the original copyright information with Keyl, and then divide the encrypted information into $n$ shares without size expansion, only one share is selected randomly and encrypted with Key2 as watermark information.

2. Decode the $3 \mathrm{D}$ videos stream to obtain the $B_{3}$ frames and $b_{4}$ frames data, where the $B_{3}$ frames are located in left view.

3. Analyze the reference relationship between $B_{3}$ and $b_{4}$ to find the locations in the $B_{3}$ frame that do not referenced by $b_{4}$ frame. These locations that in $B_{3}$ frame and all the $b_{4}$ frames are used to watermarking.

4. Use method 1 and method 2 to embed the watermark into the candidate locations by combining with matrix embedding based on Hamming codes. Key3 is used to generate the parity check matrix $\boldsymbol{H}$ of the Hamming codes. Key4 is used to determine the embedding ratio $p=k / n$, where $n=2^{k}-1$.

5. Entropy codes the watermarked $B_{3}$ frames and $b_{4}$ frames to form the final watermarked $3 \mathrm{D}$ videos stream. 


\section{Experiment Results and Conclusion}

The proposed watermarking scheme has been implemented in the H.264/MVC reference software version JM-18.6. Eight well-known standard 3D videos sequences (i.e., Akko\&kayo, Ballroom, Crowd, Exit, Flamenco, Objects, Race, and Vassar) are used for simulation. The GOP size is chosen as 8, and its coding structure is set as shown in Fig.1.

Table II Preformation Comparisons

\begin{tabular}{|c|c|c|c|c|}
\hline $\begin{array}{c}\text { 3D } \\
\text { Video }\end{array}$ & Measures & [17] & [19] & Proposed \\
\hline \multirow{3}{*}{$\begin{array}{c}\text { Akko } \\
\text { \&kayo }\end{array}$} & SSIM & 0.9941 & 0.9931 & 0.9935 \\
\hline & $\mathrm{C}$ (bit) & 32 & 106 & 155 \\
\hline & $\mathrm{CC}(\%)$ & 0.97 & 0.92 & 1 \\
\hline \multirow{3}{*}{ Ballroom } & SSIM & 0.9848 & 0.9843 & 0.9845 \\
\hline & $\mathrm{C}$ (bit) & 65 & 219 & 307 \\
\hline & $\mathrm{CC}(\%)$ & 0.96 & 0.92 & 1 \\
\hline \multirow{3}{*}{ Crowd } & SSIM & 0.9735 & 0.9733 & 0.9734 \\
\hline & $\mathrm{C}$ (bit) & 128 & 296 & 345 \\
\hline & $\mathrm{CC}(\%)$ & 0.98 & 0.93 & 1 \\
\hline \multirow{3}{*}{ Exit } & SSIM & 0.9936 & 0.9932 & .9934 \\
\hline & $\mathrm{C}$ (bit) & 95 & 253 & 314 \\
\hline & $\mathrm{CC}(\%)$ & 0.98 & 0.91 & 1 \\
\hline \multirow{3}{*}{ Flamenco } & SSIM & 0.9926 & 0.9923 & 0.9925 \\
\hline & $\mathrm{C}$ (bit) & 111 & 305 & 379 \\
\hline & $\mathrm{CC}(\%)$ & 0.97 & 0.92 & 1 \\
\hline \multirow{3}{*}{ Objects } & SSIM & 0.9941 & 0.9931 & 0.9936 \\
\hline & $\mathrm{C}$ (bit) & 67 & 263 & 324 \\
\hline & $\mathrm{CC}(\%)$ & 0.96 & 0.92 & 1 \\
\hline \multirow{3}{*}{ Race } & SSIM & 0.9768 & 0.9763 & 0.9766 \\
\hline & C (bit) & 157 & 321 & 378 \\
\hline & $\mathrm{CC}(\%)$ & 0.96 & 0.91 & 1 \\
\hline \multirow{3}{*}{ Vassar } & SSIM & 0.9739 & 0.9734 & 0.9739 \\
\hline & $\mathrm{C}$ (bit) & 201 & 329 & 382 \\
\hline & $\mathrm{CC}(\%)$ & 0.98 & 0.92 & 1 \\
\hline
\end{tabular}

Video quality, embedding capacity and computation complexity are considered to evaluate the proposed algorithm. Since PSNR (Peak Signal to Noise Ratio) does not perfectly correlate with a perceived visual quality dui to nonlinear behavior of human visual system, SSIM (Structure Similarity) [26] is adopted to evaluate the perceptual video quality in this paper. The SSIM index lies in the range between 0 and 1 , where 1 indicate the reference image is identical than the target image. In order to evaluate the real-time performance to the proposed scheme, we use $C C=T / T_{\mathrm{P}}$ to measure the computation complexity, where $T$ represent the total time consumed by the corresponding scheme, $T_{\mathrm{P}}$ is the time consumed by the proposed scheme. Watermarking capacity $C$ (bit) is used to comparison also.

The experimental results are shown in table II .It is clear that the proposed scheme has achieved greater watermarking capacity with lower degradation in SSIM than other exiting schemes. This is benefited from the more embedding locations and more efficient embedding method. One of the drawbacks of the proposed scheme is more time-consuming than other schemes as it have to analyze the reference relationship to $B_{3}$ frames. How to reduce the computational complexity is our future's work.

\section{Acknowledgment}

The work described in this paper was supported by the National Natural Science Foundation of China under Grant (61271270, U1301257, 61311140262). National Key Technology R\&D Program of the Ministry of Science and Technology under Grant No. 2012BAH67F01, Zhejiang Provincial 
Natural Science Foundation of China under Grant No. LY14F010004, and Scientific Research Foundation of Ningbo University under Grant No. XYL12001. The authors are indebted to Dr. Zhang Leo $\mathrm{Yu}$ of the Electronic Engineering Department of City University of Hong Kong for his constructive comments.

\section{References}

[1] Shao F, Lin W S, Gu S B, Jiang G Y, and Srikanthan, T. "Perceptual full-reference quality assessment of stereoscopic images by considering binocular visual characteristics," IEEE Transation on Image Processing, 2013, 22(5): 1940-1953.

[2] Wang X, Kwong S, Yuan H, Zhang Y, and Pan z, "View synthesis distortion model based frame level rate control optimization for multiview depth video coding," Signal Processing, 2014, in press.

[3] Zhang Z, Zhu Z, and Xi L, "Novel scheme for watermarking stereo video," International Journal of Nonlinear Science, 2007, 3(1): 74-80.

[4] Chammem A, Mitrea M, and Prêteux F, "Stereoscopic video watermarking: a comparative study". annals of telecommunications-annales des télécommunications, 2013, 68(11-12): 673-690.

[5] Yu M, Wang J,Jiang G Y, Peng Z J, Shao F, and Luo T. "New fragile watermarking method for stereo image authentication with localization and recovery", AEU-International Journal of Electronics and Communications, 2014.

[6] Ou Z H, and Chen L H. "A robust watermarking method for stereo-pair images based on unmatched block bitmap". Multimedia Tools and Applications, 2015: 1-22.

[7] Ho Y S, and Oh K J, "Overview of multi-view video coding", Systems, Signals and Image Processing, and 6th EURASIP Conference focused on Speech and Image Processing, Multimedia Communications and Services. 14th International Workshop on. IEEE, 2007: 5-12.

[8] Merkle P, Smolic A, Mueller K, and Wiegand T. "Efficient prediction structures for multiview video coding”. IEEE Transactions on Circuits System Video Technology, 2007, 17(11):1461-1473.

[9] Tew Y, and Wong K. "An overview of information hiding in H.264/AVC compressed video", IEEE Transactions on Circuits and Systems for Video Technology, 2014, 24(2):305-319.

[10] Mansouri A, Aznaveh A M, Torkamani-Azar F, and Kurugollu F. "A low complexity video watermarking in H. 264 compressed domain", IEEE Transactions on Information Forensics and Security, 2010, 5(4): 649-657.

[11] Li J, Liu H, Huang J, and Shi Y Q. "Reference index-based H. 264 video watermarking scheme", ACM Transactions on Multimedia Computing, Communications, and Applications (TOMCCAP), 2012, 8(2S): 33.

[12] Shahid Z, Chaumont M, and Puece W. "H.264/AVC video watermarking for active fingerprinting based on Tardos code", Signal, Image and Video Processing, 2013, 7(4): 679-694.

[13] Huo W, Zhu Y, and Chen H. "A controllable error-drift elimination scheme for watermarking algorithm in H.264/AVC Stream”. IEEE Signal Processing Letters, 2011, 18(9): 535-538.

[14] Zhang L W, Zhu Y S, and Bu L W. "A fast watermarking algorithm with low complexity error compensation in H.264/AVC bit-Stream", Acta Scientiarum Naturalium Universitatis Pekinensis, 2013, 49 (2): 227-233. (in Chinese)

[15] Lin T J, Chunk K L, Chang P C, Huang Y H, Liao H Y M, and Fang C Y. "An improved DCT-based perturbation scheme for high capacity data hiding in H. 264/AVC intra frames", Journal of Systems and Software, 2013, 86(3): 604-614. 
[16] Chen W, Shahid Z, and Stutz T. "Robust drift-free bitrate preserving H.264 watermarking", Multimedia Systems, 2014, 20(2):179-193.

[17] Ma X, Li Z, and Tu H. "A data hiding algorithm for H.264/AVC video stream without intraframe distortion drift", IEEE Transactions on Circuits and Systems for Video Technology, 2010, 20(10):1320-1325.

[18] Song G, Li Z, and Zhao J. "A reversible video steganography algorithm for MVC based on motion vector", Multimedia Tools and Applications, 2013: 1-24.

[19] Song G, Li Z, and Zhao J. "A Data Hiding Algorithm for 3D Videos Based on Inter-MBs". Intelligent Computing Theory. Springer International Publishing, 2014: 541-552

[20] Song G, Li Z, and Zhao J. "A video steganography algorithm for MVC without distortion drift”. IEEE International Conference on Audio, Language and Image Processing (ICALIP), 2014: 738-742.

[21] Zhang Y, Jiang G Y, and Yu M. "Adaptive multiview video coding scheme based on spatiotemporal correlation analyses”, ETRI journal, 2009, 31(2): 151-161.

[22] Shamir A. "How to share a secret", Communications of the ACM, 1979, 22(11): 612-613.

[23] Crandall R. "Some notes on steganography”, Posted on steganography mailing list, 1998.

[24] Munuera C. "Steganography and error-correcting codes", Signal Processing, 2007, 87(6): 1528-1533.

[25] Chang P C, Chung K L, and Chen J J, “A DCT/DST-based error propagation-free data hiding algorithm for HEVC intra-coded frames", Journal of Visual Communication and Image Representation, 2014, 25(2): 239-253.

[26] Wang Z, Bovik A C, and Sheikh H R, "Image quality assessment: from error visibility to structural similarity", IEEE Transactions on Image Processing, 2004, 13(4): 600-612. 